\title{
Changes in waist circumference and risk of all-cause and CVD mortality: results from the European Prospective Investigation into Cancer in Norfolk (EPIC-Norfolk) cohort study
}

Angela A. Mulligan ${ }^{1,2^{*}}$ (D), Marleen A. H. Lentjes ${ }^{1}$, Robert N. Luben ${ }^{1}$, Nicholas J. Wareham ${ }^{1,3}$ and Kay-Tee Khaw ${ }^{1,4}$

\begin{abstract}
Background: Measures of abdominal adiposity are strongly associated with all-cause mortality and cardiovascular disease (CVD). However, data are limited and conflicting regarding the consequences of changes in body fat distribution. The main aims of this paper are to investigate the association between changes in waist circumference (WC) and all-cause and CVD mortality and to examine these changes in relation to concurrent changes in weight.

Methods: The European Prospective Investigation into Cancer and Nutrition (EPIC-Norfolk) study recruited 25,639 participants between 1993 and 1997, aged 39-79, a number of whom also attended a second examination (19982000), and were followed up to 2016 for mortality. Participants were eligible for inclusion if they had WC, weight and height measurements at both time-points; those with a self-reported history of CVD or cancer, body mass index $<18.5 \mathrm{~kg} / \mathrm{m} 2$ or missing data on covariates were excluded, leaving 12,337 participants for analyses. The median (IQR) follow-up time was $16.4(15.7,17.2)$ years. Hazard Ratios (HRs) for all-cause (2866 deaths) and CVD mortality (822 deaths), by categories of WC change, were determined using Cox proportional hazards analyses.

Results: After multivariable adjustment, the HRs (95\% Cls) for all-cause mortality for men and women with a WC gain (WCG) $>5 \mathrm{~cm}$ were $1.51(1.29-1.75)$ and $1.25(1.06-1.46)$ respectively. For CVD mortality in men and women with a WCG $>5 \mathrm{~cm}$, the HRs were $1.84(1.39-2.43)$ and $1.15(0.85-1.55)$ respectively. In analyses of concurrent changes in WC and weight, the greatest risk (HRs) (95\% Cls) in men occurred with weight loss and WCG: 1.80 (1.132.86) for all-cause and 2.22 (1.03-4.82) for CVD mortality. In women, the greatest risk for both all-cause (HR 1.50 (1.16-1.95)) and CVD mortality (HR $1.81(1.15-2.85))$ was observed in those with weight loss and maintenance of WC (WCM).

Conclusions: Objectively measured WCG $>5 \mathrm{~cm}$, was associated with subsequent higher total mortality risk and higher CVD mortality risk in men. Interventions focusing on preventing increase in central adiposity rather than lowering weight per se in later life may potentially have greater health benefits.
\end{abstract}

Keywords: Waist circumference change, Weight change, All-cause mortality, CVD mortality, EPIC-Norfolk

\footnotetext{
* Correspondence: angela.mulligan@phpc.cam.ac.uk; angela.mulligan@mrcepid.cam.ac.uk

'European Prospective Investigation into Cancer and Nutrition, Department of Public Health and Primary Care, Strangeways Research Laboratory, University of Cambridge, Worts Causeway, Cambridge, UK

${ }^{2}$ European Prospective Investigation into Cancer and Nutrition, MRC Epidemiology Unit, Cambridge Biomedical Campus, University of Cambridge, Cambridge, UK

Full list of author information is available at the end of the article
}

(c) The Author(s). 2019 Open Access This article is distributed under the terms of the Creative Commons Attribution 4.0 International License (http://creativecommons.org/licenses/by/4.0/), which permits unrestricted use, distribution, and reproduction in any medium, provided you give appropriate credit to the original author(s) and the source, provide a link to the Creative Commons license, and indicate if changes were made. The Creative Commons Public Domain Dedication waiver (http://creativecommons.org/publicdomain/zero/1.0/) applies to the data made available in this article, unless otherwise stated. 


\section{Background}

Obesity is a major risk factor for many chronic diseases, such as diabetes, cardiovascular disease (CVD) and certain cancer types, as well as mortality [1-3], and is commonly defined using body mass index (BMI) [4]. However, BMI does not take into account the distribution of the fat mass, which is of particular importance in older individuals, as the distribution of body fat changes with age [5]. Waist circumference (WC) strongly correlates with abdominal obesity and is a commonly used clinical measure of body fat distribution $[6,7]$. Studies have shown that WC is associated with CVD risk [8-10] and CVD mortality [11]. WC has also been positively associated with all-cause mortality in a number of studies [12-18] with a few exceptions [19, 20]. The presence of central or abdominal obesity, as defined by a high WC, is one of five components used in the clinical diagnosis of the metabolic syndrome [21], which is associated with an increased risk of developing type 2 diabetes, CVD and subsequent mortality [22].

However, there are limited and conflicting data available regarding the consequences of changes in body fat distribution. A number of studies have found a gain in WC (WCG) to be predictive of subsequent all-cause and CVD mortality [23, 24] but a prospective cohort study found that WC loss (WCL) was associated with an increased risk of all-cause mortality, particularly for older adults [25]. However, no association was found between changes in WC and mortality in Swedish women [26], elderly Brazilians [27] or Iranian men [28].

Data pooled from 15 prospective studies that included 258,114 individuals, has found that a $1 \mathrm{~cm}$ increase in WC is associated with a $2 \%$ increased risk of future CVD [29]. In England, among women, the mean WC increased from $81.7 \mathrm{~cm}$ in 1993 to $88.1 \mathrm{~cm}$ in 2016, and the percentage with a very high WC $(>88 \mathrm{~cm})$ rose from 26 to $46 \%$; among men, the mean WC increased from $93.2 \mathrm{~cm}$ in 1993 to $97.0 \mathrm{~cm}$ in 2016, and the percentage with a very high WC (>102 cm) increased from 20 to 34\% [30].

We have recently reported that objectively measured weight loss (WTL), but not weight gain (WTG), was associated with subsequent higher mortality in healthy, middleaged and elderly community-dwelling men and women, compared to those who maintained their weight (WTM) [31]. The main aim of this paper is therefore to investigate the association between changes in WC and all-cause and CVD mortality. A secondary aim is to examine these changes in relation to concurrent changes in weight as previous studies have tended to focus on either changes in WC or weight but not changes in both concurrently.

\section{Methods}

\section{Study design}

The European Prospective Investigation Into Cancer and Nutrition (EPIC) is large diet and cancer cohort [32].
The EPIC-Norfolk study participants, aged between 39 and 79 years, were recruited from 35 General Practitioners' surgeries in the Norfolk area of East Anglia, from 1993 to 1997 [33]. General practice age sex registers act as a population sampling frame as practically all of the UK the population are registered with a general practice through the National Health Service. Many of the characteristics of this cohort at baseline were comparable to the UK national population, including age, sex and anthropometry measurements but this cohort had a lower proportion of current smokers [34].

Ethical approval for the study was given by the Norwich District Health Authority Ethics Committee and all participants gave written, informed consent.

\section{Main exposure: change in waist circumference}

Of those who consented to take part in the study $(N=$ 30,445), 25,639 attended a baseline health examination (1HE) (1993-1997) and 15,786 attended a second health examination (2HE) (1998-2000).

Trained nurses measured participants' weight and height, with participants wearing light clothing and without shoes. Weight was measured to the nearest $100 \mathrm{~g}$ using digital scales (Salter, UK). Height was measured to the nearest $0.1 \mathrm{~cm}$ using a free-standing stadiometer. A D-loop non-stretch fibreglass tape was used to measure WC, which was measured at the smallest circumference between the ribs and iliac crest to the nearest $0.1 \mathrm{~cm}$ while the participant was standing erect, with the abdomen relaxed, the arms at the side, the feet together and at the end of a normal expiration, without the tape compressing the skin. The measurement was taken at the level of the umbilicus where there was no natural waistline. Body mass index (BMI) is defined as the body mass (weight) divided by the square of the height, and is expressed in $\mathrm{kg} / \mathrm{m} 2$, and is commonly used to categorise individuals as underweight $(<18.5 \mathrm{~kg} / \mathrm{m} 2)$, normal weight ( $\geq 18.5$ to $<25 \mathrm{~kg} / \mathrm{m} 2$ ), overweight ( $\geq 25$ to $30 \mathrm{~kg}$ / $\mathrm{m} 2)$, or obese ( $\geq 30 \mathrm{~kg} / \mathrm{m} 2)$ [4].

Absolute change in WC $(\mathrm{cm})$ was calculated as $\mathrm{WC}$ measured at $2 \mathrm{HE}$ minus $\mathrm{WC}$ at $1 \mathrm{HE}$. Absolute weight change $(\mathrm{kg})$ was calculated as weight measured at $2 \mathrm{HE}$ minus weight measured at $1 \mathrm{HE}$. Participants were assigned to one of five WC change categories: $>5 \mathrm{~cm}$ loss, $>2.5$ to $\leq 5 \mathrm{~cm}$ loss, $\leq 2.5 \mathrm{~cm}$ loss or gain ('maintenance', including zero change; considered the reference category), $>2.5$ to $\leq 5 \mathrm{~cm}$ gain, $>5 \mathrm{~cm}$ gain. Annual WC change was calculated from the absolute difference in WC, divided by the participants' time lapse between the health examinations $(\mathrm{cm} / \mathrm{y})$.

In order to study the possible association between changes in WC and changes in weight, participants were assigned to one of nine categories, based on a loss of > $2.5 \mathrm{~cm}$ or $2.5 \mathrm{~kg}$, within $2.5 \mathrm{~cm}$ or $2.5 \mathrm{~kg}$ loss or gain 
(maintenance), or a gain of $>2.5 \mathrm{~cm}$ or $2.5 \mathrm{~kg}$ respectively. The nine categories were as follows: WTM (weight maintenance) and WCM (WC maintenance) (reference category); WTL (weight loss) and WCL (WC loss); WTL and WCM; WTL and WCG (WC gain); WTM and WCL; WTM and WCG; WTG (weight gain) and WCL; WTG and WCM; and WTG and WCG.

\section{Participant selection}

Measurements of WC, weight and height measurements at both time-points were essential for participant inclusion $(N=15,010)$. Participants were excluded from analyses if they were categorised as underweight $(\mathrm{BMI}<$ $18.5 \mathrm{~kg} / \mathrm{m}^{2}$ ) or who self-reported CVD or cancer at either examination $(N=2095)$; those with missing data on adjustment variables (smoking, social class, educational level, physical activity, and menopausal status in women $(N=529)$ were also excluded. This left 12,337 participants for analyses (Fig. 1).

\section{Adjustment variables}

Data from two self-administered Health and Lifestyle Questionnaires (HLQ1 and HLQ2), at 1HE and 2HE respectively, were used to create categories of a number of variables. Current smokers were defined as those currently smoking cigarettes, former smoker as being a smoker previously and non-smokers were those who never smoked (derived from HLQ1 and HLQ2). Self-reported physical activity (derived from HLQ1) was classified into four levels, using both occupational and leisure activity: inactive, moderately inactive,

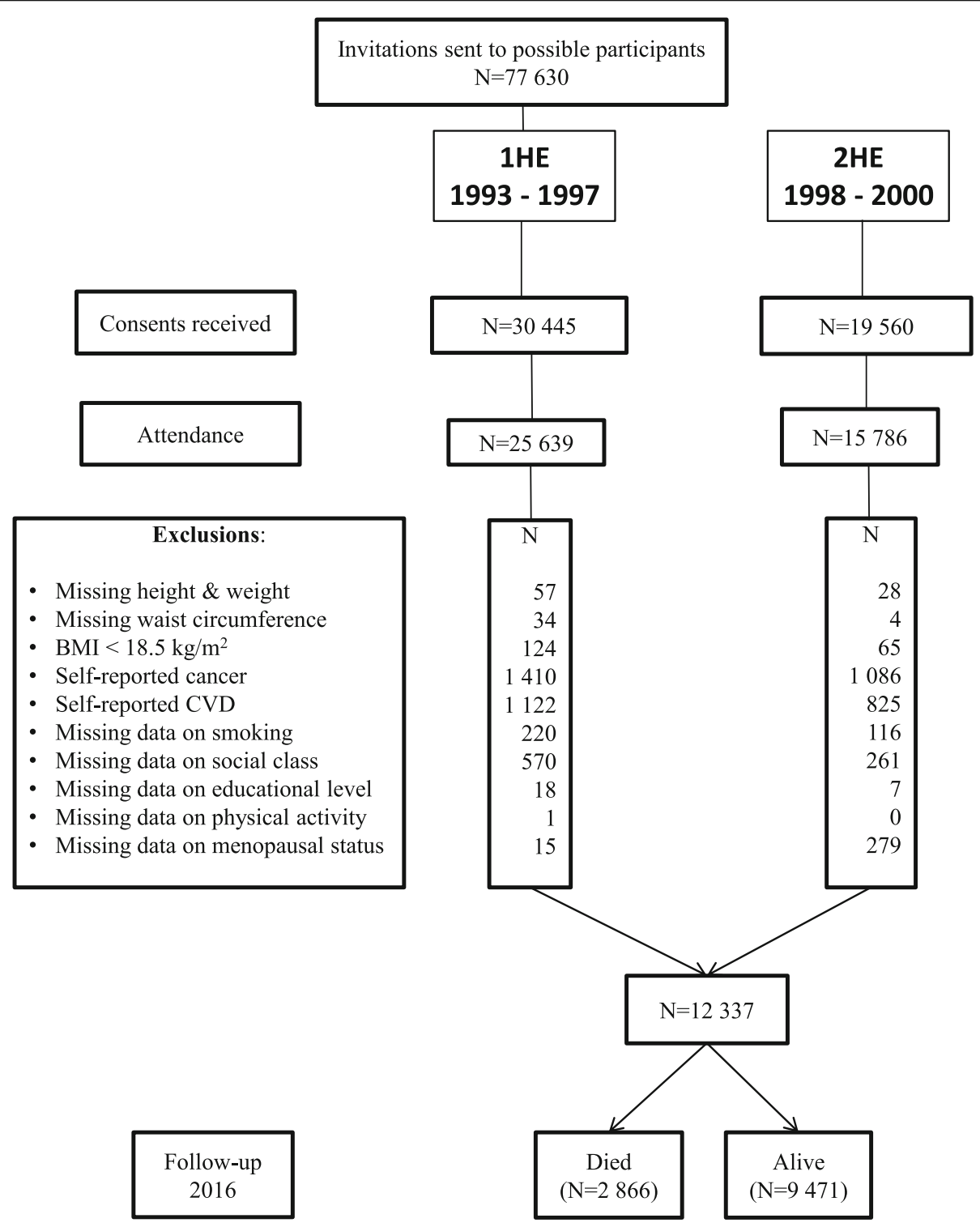

Fig. 1 Study population and sample size eligible for mortality analyses 
moderately active and active [35, 36]. Social class at $1 \mathrm{HE}$ was defined according to the Registrar General's occupation-based classification scheme and categorised into the following six groups: professional, managerial and technical, non-manual skilled, manual skilled, partly skilled and unskilled [37]; for our analyses, these categories were combined to create 2 categories, non-manual (professional, managerial and technical, non-manual skilled) and manual (manual skilled, partly skilled and unskilled). Educational status at $1 \mathrm{HE}$ was categorised into four groups: degree or equivalent, $A$ level or equivalent, $O$ level or equivalent and less than $\mathrm{O}$ level or no qualification, corresponding to levels within the International Standard Classification of Education 1997 [38]. In our analyses, those with an educational level of $\mathrm{O}$ level and above were combined into one category. Menopausal status was categorised as premenopausal, early perimenopausal ( $<1$ year), late perimenopausal ( $1-5$ years) or postmenopausal ( $>5$ years). Included adjustment variables were measured with HLQ1, with the exceptions of smoking status and menopausal status, which were measured with HLQ2. Participants were asked about their medical histories at both time-points. The diagnosis of chronic diseases such as heart attack, stroke and cancer were recorded as present when participants answered "yes" to question "Has a doctor ever told you that you have any of the following conditions?"

\section{Endpoints}

All fatal events occurring between 1998 and 31st March 2016 were captured by the Office of National Statistics, United Kingdom. Death certificates were coded by nosologists according to the International Classification of Diseases (ICD). An underlying cause of death from cancer or CVD was defined by using the following ICD codes: cancer death (ICD9, 140-208 or ICD10 C00C97), or CVD death (ICD9 400-438 or ICD10 I10-I79).

\section{Statistical analyses}

Characteristics of the study population were summarised by WC change category, for continuous variables (mean and SD) and categorical variables (frequency and percentage). The follow-up time began at the $2 \mathrm{HE}$ and formed the underlying time variable; median (IQR) follow-up time was $16.4(15.7,17.2)$ years. Participants who died were censored at their date of death and those who did not die were censored at the end of follow-up (31st March 2016). Three Cox proportional hazards models were used to determine Hazard Ratios (HRs) for all-cause and CVD mortality by WC change category for men and women separately: age (continuous variable), (model 1); including BMI (continuous variable), WC (continuous variable), physical activity, social class, educational level, smoking (2HE) and menopausal status $(2 \mathrm{HE})$ in women (model 2); and including difference in weight (continuous variable) (model 3). The proportional hazards' assumption was tested by including time interaction variables in the Cox regression models and we found that age violated our test $(P<0.0001)$. However, when the time interaction for age was included, only minimal changes to the HRs of WC change were observed, so results are shown with age adjustment alone. We also examined HRs by WC change category, separately in men and women, in subgroups, stratified by age, WC, smoking status, BMI, physical activity, educational level, social class, menopausal status in women, and after the exclusion of individuals who died within 3 or 5 years of the $2 \mathrm{HE}$. The data were analysed using Stata 14 (STATA Corp., Texas, USA).

\section{Results}

\section{Description of the cohort}

Over a 3.5 year period, men had a mean WC and weight increase of $0.83 \mathrm{~cm}$ (SD 5.19) and $1.29 \mathrm{~kg}$ (SD 3.61) respectively; similar mean increases of $0.81 \mathrm{~cm}$ (SD 5.60) in WC and $1.38 \mathrm{~kg}$ (SD 4.14) in weight were found in women. The baseline characteristics of those who attended both $1 \mathrm{HE}$ and $2 \mathrm{HE}$, before and after exclusions were applied were similar (Additional file 1: Table S1). However, the prevalence of self-reported CVD and the percentages of current smokers and physically inactive participants were higher in those who attended the $1 \mathrm{HE}$ only, compared to those who also attended the 2HE. Also, the percentage of deaths that occurred was lower in both men and women, after exclusion criteria were applied, with the highest percentage found in those who only attended the $1 \mathrm{HE}$.

Table 1 describes the characteristics of men and women, by WC change category. WCM was observed in $40 \%$ of men and $38 \%$ of women. Participants with the greatest WCL were, on average, older and had the largest WC, weight and BMI at $1 \mathrm{HE}$. Men with the greatest WCG were more likely to have a smaller WC at $1 \mathrm{HE}$, whereas women with the greatest WCG had a similar WC at 1HE, compared to those in the WCM category. Current smokers at $2 \mathrm{HE}$ were more likely to have a WCL whereas former smokers, a WCG. Male nonmanual workers were more likely to have a $\mathrm{WCL}>5 \mathrm{~cm}$ whereas male manual workers were more likely to have a WCG $>5 \mathrm{~cm}$. Participants with no qualifications were more likely to have a WCG $>5 \mathrm{~cm}$. A greater percentage of deaths occurred in those with a WCL $>5 \mathrm{~cm}$.

\section{Main analyses: total and CVD mortality}

At the end of a median (IQR) follow-up time of 16.4 $(15.7,17.2)$ years, 1551 deaths in men and 1315 deaths in women were recorded, 440 and 382 deaths from CVD, respectively. Total and CVD mortality HRs for men and women by WC change category are shown in 
Table 1 Characteristics at 1st and 2nd health examinations of 5469 men and 6868 women, stratified by categories of change in waist circumference

\begin{tabular}{|c|c|c|c|c|c|c|c|c|c|c|c|c|}
\hline \multirow{4}{*}{$\begin{array}{l} \\
M E N, N(\text { row \%) } \\
\text { WC at } 1 \mathrm{HE}(\mathrm{cm})\end{array}$} & \multicolumn{12}{|c|}{ Categories of change in waist circumference (WC) $(\mathrm{cm})$} \\
\hline & \multicolumn{2}{|c|}{ Loss $>5 \mathrm{~cm}$} & \multicolumn{2}{|c|}{ Loss $>2.5$ and $\leq 5 \mathrm{~cm}$} & \multicolumn{2}{|c|}{ Loss or gain $\leq 2.5 \mathrm{~cm}$} & \multicolumn{2}{|c|}{ Gain $>2.5$ and $\leq 5 \mathrm{~cm}$} & \multicolumn{2}{|c|}{ Gain $>5 \mathrm{~cm}$} & \multicolumn{2}{|l|}{ All } \\
\hline & \multicolumn{2}{|c|}{$641(11.7)$} & \multicolumn{2}{|c|}{$656(12.0)$} & \multicolumn{2}{|c|}{$2211(40.4)$} & \multicolumn{2}{|c|}{$926(16.9)$} & \multicolumn{2}{|c|}{$1035(18.9)$} & 5469 & $100.0 \%$ \\
\hline & 99.0 & 9.2 & 96.8 & 9.1 & 95.3 & 8.9 & 93.8 & 8.6 & 92.4 & 9.7 & 95.1 & 9.3 \\
\hline WC at $2 \mathrm{HE}(\mathrm{cm})$ & 91.0 & 9.2 & 93.2 & 9.1 & 95.4 & 8.9 & 97.6 & 8.6 & 100.5 & 10.0 & 96.0 & 9.6 \\
\hline WC change $(\mathrm{cm})$ & -8.0 & 3.1 & -3.7 & 0.7 & 0.1 & 1.4 & 3.8 & 0.7 & 8.1 & 2.9 & 0.8 & 5.2 \\
\hline $\begin{array}{l}\text { Annual WC } \\
\text { change }(\mathrm{cm})\end{array}$ & -2.3 & 1.0 & -1.1 & 0.3 & 0.0 & 0.4 & 1.1 & 0.3 & 2.2 & 0.9 & 0.2 & 1.5 \\
\hline Age at $1 \mathrm{HE}$ (years) & 59.6 & 9.3 & 59.7 & 9.0 & 58.8 & 8.9 & 58.7 & 8.9 & 58.6 & 8.7 & 58.9 & 8.9 \\
\hline Age at $2 \mathrm{HE}$ (years) & 62.8 & 9.4 & 62.8 & 9.1 & 61.9 & 9.0 & 61.9 & 9.0 & 61.8 & 8.7 & 62.1 & 9.0 \\
\hline Age change (years) & 3.2 & 0.8 & 3.1 & 0.8 & 3.1 & 0.8 & 3.2 & 0.8 & 3.3 & 0.8 & 3.2 & 0.8 \\
\hline Weight at $1 \mathrm{HE}(\mathrm{kg})$ & 81.7 & 10.7 & 80.4 & 10.9 & 80.0 & 10.8 & 79.8 & 10.2 & 80.2 & 11.7 & 80.2 & 10.9 \\
\hline Weight at 2HE (kg) & 79.0 & 10.5 & 79.9 & 11.0 & 81.0 & 11.2 & 82.4 & 10.7 & 84.4 & 12.8 & 81.5 & 11.5 \\
\hline Weight change (kg) & -2.7 & 3.6 & -0.5 & 2.9 & 1.1 & 2.6 & 2.6 & 2.7 & 4.2 & 3.5 & 1.3 & 3.6 \\
\hline BMl at $1 \mathrm{HE}\left(\mathrm{kg} / \mathrm{m}^{2}\right)$ & 26.8 & 3.2 & 26.5 & 3.1 & 26.3 & 3.1 & 26.2 & 3.0 & 26.3 & 3.3 & 26.3 & 3.1 \\
\hline $\mathrm{BMI}$ at $2 \mathrm{HE}\left(\mathrm{kg} / \mathrm{m}^{2}\right)$ & 26.0 & 3.1 & 26.4 & 3.2 & 26.7 & 3.2 & 27.1 & 3.1 & 27.8 & 3.6 & 26.9 & 3.3 \\
\hline BMl change $\left(\mathrm{kg} / \mathrm{m}^{2}\right)$ & -0.8 & 1.2 & -0.1 & 0.9 & 0.4 & 0.9 & 0.9 & 0.9 & 1.5 & 1.2 & 0.5 & 1.2 \\
\hline \multicolumn{13}{|l|}{ Education - 1HE } \\
\hline None & 172 & 26.8 & 164 & 25.0 & 573 & 25.9 & 248 & 26.8 & 287 & 27.7 & 1444 & $26.4 \%$ \\
\hline O level and above & 469 & 73.2 & 492 & 75.0 & 1638 & 74.1 & 678 & 73.2 & 748 & 72.3 & 4025 & $73.6 \%$ \\
\hline \multicolumn{13}{|l|}{ Social class - 1HE } \\
\hline Non-manual & 412 & 64.3 & 414 & 63.1 & 1352 & 61.2 & 585 & 63.2 & 632 & 61.1 & 3395 & $62.1 \%$ \\
\hline Manual & 229 & 35.7 & 242 & 36.9 & 859 & 38.8 & 341 & 36.8 & 403 & 38.9 & 2074 & $37.9 \%$ \\
\hline \multicolumn{13}{|l|}{ Smoking status - 1HE } \\
\hline Current & 66 & 10.3 & 60 & 9.2 & 185 & 8.4 & 86 & 9.3 & 110 & 10.6 & 507 & $9.3 \%$ \\
\hline Former & 321 & 50.1 & 341 & 52.0 & 1200 & 54.3 & 477 & 51.5 & 559 & 54.0 & 2898 & $53.0 \%$ \\
\hline Never & 254 & 39.6 & 255 & 38.9 & 826 & 37.4 & 363 & 39.2 & 366 & 35.4 & 2064 & $37.7 \%$ \\
\hline \multicolumn{13}{|l|}{ Smoking status - $2 \mathrm{HE}$} \\
\hline Current & 67 & 10.5 & 59 & 9.0 & 166 & 7.5 & 65 & 7.0 & 81 & 7.8 & 438 & $8.0 \%$ \\
\hline Former & 320 & 49.9 & 342 & 52.1 & 1221 & 55.2 & 498 & 53.8 & 589 & 56.9 & 2970 & $54.3 \%$ \\
\hline Never & 254 & 39.6 & 255 & 38.9 & 824 & 37.3 & 363 & 39.2 & 365 & 35.3 & 2061 & $37.7 \%$ \\
\hline \multicolumn{13}{|l|}{ Physical activity - $1 \mathrm{HE}$} \\
\hline Inactive & 193 & 30.1 & 160 & 24.4 & 601 & 27.2 & 233 & 25.2 & 257 & 24.8 & 1444 & $26.4 \%$ \\
\hline Moderately inactive & 163 & 25.4 & 196 & 29.9 & 529 & 23.9 & 216 & 23.3 & 249 & 24.1 & 1353 & $24.7 \%$ \\
\hline Moderately active & 156 & 24.3 & 159 & 24.2 & 541 & 24.5 & 249 & 26.9 & 272 & 26.3 & 1377 & $25.2 \%$ \\
\hline Active & 129 & 20.1 & 141 & 21.5 & 540 & 24.4 & 228 & 24.6 & 257 & 24.8 & 1295 & $23.7 \%$ \\
\hline Deaths during follow-up & 218 & 34.0 & 196 & 29.9 & 585 & 26.5 & 246 & 26.6 & 306 & 29.6 & 1551 & $28.4 \%$ \\
\hline \multicolumn{13}{|l|}{$\begin{array}{l}\text { Lost weight in } \\
\text { last } 5 \text { years }(2 \mathrm{HE})\end{array}$} \\
\hline Diet & 61 & 22.9 & 42 & 15.8 & 92 & 34.6 & 28 & 10.5 & 43 & 16.2 & 266 & $4.9 \%$ \\
\hline Illness & 28 & 21.7 & 7 & 5.4 & 51 & 39.5 & 17 & 13.2 & 26 & 20.2 & 129 & $2.4 \%$ \\
\hline WOMEN, N (row \%) & \multicolumn{2}{|c|}{$873(12.7)$} & 875 & & 2596 & & 1131 & & 1393 & & 6868 & $100.0 \%$ \\
\hline WC at $1 \mathrm{HE}(\mathrm{cm})$ & 87.2 & 11.4 & 82.6 & 10.5 & 80.0 & 9.5 & 79.1 & 9.7 & 79.6 & 9.2 & 81.0 & 10.2 \\
\hline WC at $2 \mathrm{HE}(\mathrm{cm})$ & 78.8 & 10.7 & 78.9 & 10.5 & 80.1 & 9.6 & 82.8 & 9.7 & 88.0 & 10.0 & 81.9 & 10.5 \\
\hline WC change $(\mathrm{cm})$ & -8.4 & 3.5 & -3.7 & 0.8 & 0.1 & 1.4 & 3.7 & 0.7 & 8.4 & 3.3 & 0.8 & 5.6 \\
\hline Annual WC change $(\mathrm{cm})$ & -2.4 & 1.2 & -1.1 & 0.3 & 0.0 & 0.4 & 1.1 & 0.3 & 2.4 & 1.1 & 0.2 & 1.6 \\
\hline Age at $1 \mathrm{HE}$ (years) & 58.7 & 9.0 & 58.5 & 9.2 & 58.0 & 8.9 & 57.4 & 8.6 & 57.5 & 8.5 & 58.0 & 8.8 \\
\hline
\end{tabular}


Table 1 Characteristics at 1st and 2nd health examinations of 5469 men and 6868 women, stratified by categories of change in waist circumference (Continued)

\begin{tabular}{|c|c|c|c|c|c|c|c|c|c|c|c|c|}
\hline \multirow[b]{3}{*}{ Age at 2HE (years) } & \multicolumn{12}{|c|}{ Categories of change in waist circumference $(\mathrm{WC})(\mathrm{cm})$} \\
\hline & \multicolumn{2}{|c|}{ Loss $>5 \mathrm{~cm}$} & \multicolumn{2}{|c|}{ Loss $>2.5$ and $\leq 5 \mathrm{~cm}$} & \multicolumn{2}{|c|}{ Loss or gain $\leq 2.5 \mathrm{~cm}$} & \multicolumn{2}{|c|}{ Gain $>2.5$ and $\leq 5 \mathrm{~cm}$} & \multicolumn{2}{|c|}{ Gain $>5 \mathrm{~cm}$} & \multicolumn{2}{|l|}{ All } \\
\hline & 61.9 & 9.2 & 61.6 & 9.3 & 61.2 & 9.0 & 60.5 & 8.6 & 60.6 & 8.6 & 61.1 & 8.9 \\
\hline Age change (years) & 3.1 & 0.8 & 3.2 & 0.8 & 3.1 & 0.8 & 3.1 & 0.8 & 3.2 & 0.8 & 3.2 & 0.8 \\
\hline Weight at $1 \mathrm{HE}(\mathrm{kg})$ & 70.1 & 12.8 & 67.8 & 11.7 & 66.2 & 10.4 & 66.7 & 10.6 & 68.5 & 10.7 & 67.5 & 11.1 \\
\hline Weight at $2 \mathrm{HE}(\mathrm{kg})$ & 67.7 & 12.1 & 67.3 & 11.7 & 67.3 & 10.8 & 69.2 & 11.2 & 73.0 & 12.0 & 68.9 & 11.6 \\
\hline Weight change (kg) & -2.4 & 4.8 & -0.5 & 3.3 & 1.1 & 2.9 & 2.5 & 3.2 & 4.5 & 4.2 & 1.4 & 4.1 \\
\hline $\mathrm{BMI}$ at $1 \mathrm{HE}\left(\mathrm{kg} / \mathrm{m}^{2}\right)$ & 26.9 & 4.6 & 26.0 & 4.3 & 25.5 & 3.8 & 25.6 & 3.9 & 26.3 & 3.9 & 25.9 & 4.0 \\
\hline $\mathrm{BMI}$ at $2 \mathrm{HE}\left(\mathrm{kg} / \mathrm{m}^{2}\right)$ & 26.1 & 4.3 & 26.0 & 4.4 & 26.0 & 4.0 & 26.6 & 4.2 & 28.1 & 4.4 & 26.6 & 4.3 \\
\hline BMl change $\left(\mathrm{kg} / \mathrm{m}^{2}\right)$ & -0.8 & 1.8 & -0.1 & 1.2 & 0.5 & 1.1 & 1.0 & 1.2 & 1.9 & 1.6 & 0.7 & 1.6 \\
\hline \multicolumn{13}{|l|}{ Education - $1 \mathrm{HE}$} \\
\hline None & 291 & 33.3 & 321 & 36.7 & 983 & 37.9 & 402 & 35.5 & 528 & 37.9 & 2525 & $36.8 \%$ \\
\hline O level and above & 582 & 66.7 & 554 & 63.3 & 1613 & 62.1 & 729 & 64.5 & 865 & 62.1 & 4343 & $63.2 \%$ \\
\hline \multicolumn{13}{|l|}{ Social class - 1HE } \\
\hline Non-manual & 537 & 61.5 & 558 & 63.8 & 1659 & 63.9 & 727 & 64.3 & 877 & 63.0 & 4358 & $63.4 \%$ \\
\hline Manual & 336 & 38.5 & 317 & 36.2 & 937 & 36.1 & 404 & 35.7 & 516 & 37.0 & 2510 & $36.6 \%$ \\
\hline \multicolumn{13}{|l|}{ Smoking status - $1 \mathrm{HE}$} \\
\hline Current & 94 & 10.8 & 72 & 8.2 & 224 & 8.6 & 100 & 8.8 & 128 & 9.2 & 618 & $9.0 \%$ \\
\hline Former & 290 & 33.2 & 287 & 32.8 & 794 & 30.6 & 329 & 29.1 & 469 & 33.7 & 2169 & $31.6 \%$ \\
\hline Never & 489 & 56.0 & 516 & 59.0 & 1578 & 60.8 & 702 & 62.1 & 796 & 57.1 & 4081 & $59.4 \%$ \\
\hline \multicolumn{13}{|l|}{ Smoking status - $2 \mathrm{HE}$} \\
\hline Current & 88 & 10.1 & 70 & 8.0 & 206 & 7.9 & 76 & 6.7 & 105 & 7.5 & 545 & $7.9 \%$ \\
\hline Former & 297 & 34.0 & 289 & 33.0 & 814 & 31.4 & 353 & 31.2 & 494 & 35.5 & 2247 & $32.7 \%$ \\
\hline Never & 488 & 55.9 & 516 & 59.0 & 1576 & 60.7 & 702 & 62.1 & 794 & 57.0 & 4076 & $59.4 \%$ \\
\hline \multicolumn{13}{|l|}{ Physical activity - $1 \mathrm{HE}$} \\
\hline Inactive & 234 & 26.8 & 236 & 27.0 & 622 & 24.0 & 245 & 21.7 & 369 & 26.5 & 1706 & $24.8 \%$ \\
\hline Moderately inactive & 282 & 32.3 & 275 & 31.4 & 874 & 33.7 & 390 & 34.5 & 457 & 32.8 & 2278 & $33.2 \%$ \\
\hline Moderately active & 220 & 25.2 & 206 & 23.5 & 631 & 24.3 & 291 & 25.7 & 328 & 23.6 & 1676 & $24.4 \%$ \\
\hline Active & 137 & 15.7 & 158 & 18.1 & 469 & 18.1 & 205 & 18.1 & 239 & 17.2 & 1208 & $17.6 \%$ \\
\hline Deaths during follow-up & 212 & 24.3 & 181 & 20.7 & 487 & 18.8 & 176 & 15.6 & 259 & 18.6 & 1315 & $19.2 \%$ \\
\hline \multicolumn{13}{|l|}{$\begin{array}{l}\text { Menopausal } \\
\text { status (2HE) }\end{array}$} \\
\hline Pre-menopausal & 51 & 5.8 & 55 & 6.3 & 170 & 6.6 & 67 & 5.9 & 86 & 6.2 & 429 & $6.3 \%$ \\
\hline Early peri-menopausal & 26 & 3.0 & 34 & 3.9 & 80 & 3.1 & 63 & 5.6 & 48 & 3.5 & 251 & $3.7 \%$ \\
\hline Late peri-menopausal (1-5 y) & 149 & 17.1 & 168 & 19.2 & 504 & 19.4 & 213 & 18.8 & 288 & 20.7 & 1322 & $19.3 \%$ \\
\hline Post-menopausal (> 5 y) & 647 & 74.1 & 618 & 70.6 & 1842 & 71.0 & 788 & 69.7 & 971 & 69.7 & 4866 & $70.9 \%$ \\
\hline \multicolumn{13}{|l|}{$\begin{array}{l}\text { Lost weight in } \\
\text { last } 5 \text { years }(2 \mathrm{HE})\end{array}$} \\
\hline Diet & 179 & 23.6 & 108 & 14.3 & 233 & 30.8 & 94 & 12.4 & 143 & 18.9 & 757 & $11.0 \%$ \\
\hline Illness & 33 & 19.5 & 25 & 14.8 & 62 & 36.7 & 26 & 15.4 & 23 & 13.6 & 169 & $2.5 \%$ \\
\hline
\end{tabular}

Values are mean and SD or frequency and percentage

$1 H E$ 1st health examination, $2 H E$ 2nd health examination, $B M I$ body mass index

Table 2. The addition of change in weight to the model strengthened the positive WCG-mortality associations (model 3), especially in men, where the findings for CVD mortality were stronger than for all-cause mortality, in those with a WCG $>5 \mathrm{~cm}$ (HR 1.84 and 1.51 respectively). Women with a WCG $>5 \mathrm{~cm}$ had a significantly higher hazard of all-cause mortality of 1.25 (CI 1.06-1.46) compared to WCM. No significant associations were found regarding changes in WC and CVD mortality in women. No significant associations were 
Table 2 Associations between total and CVD mortality in 5469 men and 6868 women and categories of change in waist circumference

\begin{tabular}{|c|c|c|c|c|c|}
\hline & \multicolumn{5}{|c|}{ Categories of change in waist circumference (WC) $(\mathrm{cm})$} \\
\hline & Loss $>5 \mathrm{~cm}$ & Loss $>2.5$ and $\leq 5 \mathrm{~cm}$ & Loss or gain $\leq 2.5 \mathrm{~cm}$ & Gain $>2.5$ and $\leq 5 \mathrm{~cm}$ & Gain $>5 \mathrm{~cm}$ \\
\hline Men, N (\%) & $641(11.7)$ & $656(12.0)$ & $2211(40.4)$ & $926(16.9)$ & $1035(18.9)$ \\
\hline \multicolumn{6}{|l|}{ All-cause mortality } \\
\hline Number of events & $218(34.0)$ & $196(29.9)$ & $585(26.5)$ & $246(26.6)$ & $306(29.5)$ \\
\hline Model 1 & ** $1.28(1.09-1.49)$ & $1.07(0.91-1.26)$ & Ref & $1.04(0.90-1.21)$ & * $1.19(1.03-1.36)$ \\
\hline Model 2 & $1.14(0.97-1.34)$ & $1.03(0.87-1.21)$ & Ref & $1.06(0.91-1.23)$ & ** $1.26(1.10-1.46)$ \\
\hline Model 3 & $0.93(0.78-1.10)$ & $0.95(0.80-1.12)$ & Ref & $1.15(0.99-1.34)$ & $* * * 1.51(1.29-1.75)$ \\
\hline \multicolumn{6}{|l|}{ CVD mortality } \\
\hline Number of events & $64(10.0)$ & $62(9.4)$ & $153(6.9)$ & $66(7.1)$ & $95(9.2)$ \\
\hline Model 1 & * $1.42(1.06-1.90)$ & $1.28(0.95-1.72)$ & Ref & $1.08(0.81-1.44)$ & ** $1.42(1.10-1.84)$ \\
\hline Model 2 & $1.22(0.90-1.64)$ & $1.21(0.90-1.63)$ & Ref & $1.09(0.82-1.46)$ & ** $1.53(1.17-1.98)$ \\
\hline Model 3 & $0.97(0.70-1.34)$ & $1.11(0.82-1.49)$ & Ref & $1.18(0.88-1.58)$ & *** $1.84(1.39-2.43)$ \\
\hline Women, N (\%) & $873(12.7)$ & $875(12.7)$ & $2596(37.8)$ & $1131(16.5)$ & $1393(20.3)$ \\
\hline \multicolumn{6}{|l|}{ All-cause mortality } \\
\hline Number of events & $212(24.3)$ & $181(20.7)$ & $487(18.8)$ & $176(15.6)$ & $259(18.6)$ \\
\hline Model 1 & ** $1.26(1.08-1.48)$ & $1.03(0.87-1.22)$ & Ref & $0.86(0.73-1.03)$ & $1.09(0.93-1.26)$ \\
\hline Model 2 & $1.13(0.95-1.33)$ & $0.97(0.82-1.16)$ & Ref & $0.91(0.76-1.08)$ & $1.15(0.98-1.34)$ \\
\hline Model 3 & $1.03(0.87-1.24)$ & $0.93(0.78-1.10)$ & Ref & $0.95(0.79-1.13)$ & ** $1.25(1.06-1.46)$ \\
\hline \multicolumn{6}{|l|}{ CVD mortality } \\
\hline Number of events & $49(5.6)$ & $56(6.4)$ & $152(5.8)$ & $50(4.4)$ & $75(5.4)$ \\
\hline Model 1 & $0.91(0.66-1.26)$ & $0.97(0.72-1.32)$ & Ref & $0.81(0.59-1.12)$ & $1.04(0.79-1.38)$ \\
\hline Model 2 & $0.80(0.57-1.11)$ & $0.91(0.67-1.24)$ & Ref & $0.87(0.63-1.20)$ & $1.14(0.86-1.52)$ \\
\hline Model 3 & $0.77(0.54-1.09)$ & $0.89(0.65-1.22)$ & Ref & $0.87(0.63-1.21)$ & $1.15(0.85-1.55)$ \\
\hline
\end{tabular}

*** $p<0.001 ; * * 0<0.01 ;{ }^{*} p<0.05$

Associations were assessed using Cox proportional hazards regression with a median follow-up from $2 \mathrm{HE}$ of 16 years. Results are hazard ratios and $95 \%$ confidence intervals, HR $(95 \% \mathrm{Cl})$

Model 1: adjusted for age (continuous)

Model 2: Model 1 + further adjusted for BMI (continuous), waist circumference (continuous), smoking (categorical), physical activity (categorical), educational level (categorical) and social class (categorical), and menopausal status (categorical) in women

Model 3: Model $2+$ further adjusted for weight change (continuous)

$2 H E$ 2nd health examination, CVD cardiovascular disease, $B M I$ body mass index

found for WCL and total or CVD mortality in the multivariate adjusted models, in either men or women.

\section{Subgroup analyses}

HRs for all-cause mortality in men and women, adjusted as per model 3, grouped by confounder categories are shown in Tables 3 and 4 respectively. Participants with a WCG $>5$ $\mathrm{cm}$ had a higher HR than those in the WCM category, irrespective of their WC categorisation at 1HE, although stronger associations were more evident in men. Men who had a WCG $>5 \mathrm{~cm}$ had significantly higher HRs, irrespective of whether they were $<65$ years (HR 1.78 CI $1.40-2.26$ ) or $>$ 65 years (HR 1.38 (CI 1.13-1.68) of age at $1 \mathrm{HE}$ (Table 3 ) although a significant association was only found among women > 65 years (HR 1.35 (CI 1.10-1.67) (Table 4). Higher hazards were observed for participants with a WCG $>5 \mathrm{~cm}$, irrespective of smoking status, with the exception of women who were former smokers, where a WCG between 2.5 and $5 \mathrm{~cm}$ had a significantly lower HR of 0.62 (CI $0.45-0.86$ ) (Table 4). Irrespective of BMI, physical activity, educational level or social class categorisation at HE1, WCG $>5 \mathrm{~cm}$ was associated with a higher HR in both men and women (Tables 3 and 4 respectively). Almost 71\% of women were classified as postmenopausal and a higher hazard was found in those with a WCG $>5 \mathrm{~cm}$ (Table 4). Even after excluding participants who died within 3 or 5 years of the $2 \mathrm{HE}$, participants with a WCG $>5 \mathrm{~cm}$ had significantly higher HRs for all-cause mortality, than those in the WCM category.

As significant findings were evident for CVD mortality and men, but not women, in relation to changes in WC, we further explored these in subgroups, stratified by potential confounding characteristics, adjusted as per model 3 (Additional file 1: Table S2). Generally, a higher hazard was evident for all values of the confounding 
Table 3 Cox multivariable-adjusted HRs after 16 years of follow-up for all-cause mortality in 5469 men. Results are given for stratified variables by WC change category

\begin{tabular}{|c|c|c|c|c|c|c|c|c|c|}
\hline & & & & & \multicolumn{5}{|c|}{ Categories of change in waist circumference $(\mathrm{WC})(\mathrm{cm})$} \\
\hline & & & & & loss $>5 \mathrm{~cm}$ & $\begin{array}{l}\text { loss }>2.5 \\
\& \leq 5 \mathrm{~cm}\end{array}$ & $\begin{array}{l}\text { loss or gain } \\
\leq 2.5 \mathrm{~cm}\end{array}$ & $\begin{array}{l}\text { gain }>2.5 \\
\& \leq 5 \mathrm{~cm}\end{array}$ & gain $>5 \mathrm{~cm}$ \\
\hline & N & $\%$ & Deaths & $\%$ & $\mathrm{HR}(95 \% \mathrm{Cl})$ & $\mathrm{HR}(95 \% \mathrm{Cl})$ & & $\mathrm{HR}(95 \% \mathrm{Cl})$ & $\mathrm{HR}(95 \% \mathrm{Cl})$ \\
\hline All & 5469 & 100.0 & 1551 & 100.0 & $\begin{array}{l}0.93 \\
(0.78-1.10)\end{array}$ & $0.95(0.80-1.12)$ & Ref & $1.15(0.99-1.34)$ & $* * * 1.51(1.29-1.75)$ \\
\hline \multicolumn{10}{|l|}{ By WC (1HE) } \\
\hline$<94 \mathrm{~cm}$ & 2515 & 46.0 & 604 & 38.9 & $\begin{array}{l}0.93 \\
(0.67-1.28)\end{array}$ & $1.20(0.91-1.57)$ & Ref & $1.04(0.81-1.32)$ & * $1.28(1.02-1.61)$ \\
\hline$\geq 94 \& \leq 102 \mathrm{~cm}$ & 1854 & 33.9 & 520 & 33.5 & $\begin{array}{l}0.93 \\
(0.69-1.24)\end{array}$ & $0.79(0.58-1.06)$ & Ref & * $1.34(1.04-1.73)$ & $* * * 1.96(1.50-2.55)$ \\
\hline$>102 \mathrm{~cm}$ & 1100 & 20.1 & 427 & 27.5 & $\begin{array}{l}0.89 \\
(0.66-1.20)\end{array}$ & $0.90(0.67-1.21)$ & Ref & $1.20(0.88-1.65)$ & * 1.42 (1.02-1.99) \\
\hline \multicolumn{10}{|l|}{ By age (1HE) } \\
\hline$<65 y$ & 3882 & 71.0 & 573 & 36.9 & $\begin{array}{l}0.99 \\
(0.73-1.34)\end{array}$ & $0.97(0.73-1.29)$ & Ref & $1.22(0.95-1.57)$ & $* * * 1.78(1.40-2.26)$ \\
\hline$\geq 65 y$ & 1587 & 29.0 & 978 & 63.1 & $\begin{array}{l}0.91 \\
(0.74-1.13)\end{array}$ & $0.94(0.77-1.15)$ & Ref & $1.14(0.94-1.38)$ & ** $1.38(1.13-1.68)$ \\
\hline \multicolumn{10}{|l|}{$\begin{array}{l}\text { By smoking } \\
\text { status }(2 \mathrm{HC})\end{array}$} \\
\hline current & 438 & 8.0 & 170 & 11.0 & $\begin{array}{l}0.75 \\
(0.45-1.23)\end{array}$ & $0.98(0.60-1.58)$ & Ref & $1.01(0.61-1.68)$ & $* * 2.11(1.32-3.36)$ \\
\hline former & 2970 & 54.3 & 983 & 63.4 & $\begin{array}{l}0.91 \\
(0.72-1.14)\end{array}$ & $0.89(0.72-1.10)$ & Ref & $1.15(0.95-1.39)$ & $* * * 1.54(1.28-1.87)$ \\
\hline never & 2061 & 37.7 & 398 & 25.7 & $\begin{array}{l}1.17 \\
(0.84-1.63)\end{array}$ & $1.08(0.78-1.49)$ & Ref & $1.26(0.93-1.70)$ & $1.22(0.89-1.67)$ \\
\hline \multicolumn{10}{|l|}{ By BMI (1HE) } \\
\hline$\geq 18.5 \&<25$ & 1913 & 35.0 & 505 & 32.6 & $\begin{array}{l}1.03 \\
(0.75-1.41)\end{array}$ & $0.94(0.70-1.26)$ & Ref & $1.03(0.79-1.34)$ & $1.29(0.99-1.69)$ \\
\hline$\geq 25 \&<30$ & 2926 & 53.5 & 831 & 53.6 & $\begin{array}{l}0.80 \\
(0.63-1.02)\end{array}$ & $0.94(0.75-1.17)$ & Ref & $1.22(0.99-1.50)$ & ***1.69 (1.37-2.08) \\
\hline$\geq 30$ & 630 & 11.5 & 215 & 13.9 & $1.26(0.81-1.95)$ & $0.89(0.56-1.42)$ & Ref & $1.38(0.91-2.10)$ & $1.34(0.88-2.04)$ \\
\hline \multicolumn{10}{|c|}{ By physical activity (1HC) } \\
\hline inactive & 1444 & 26.4 & 545 & 35.1 & $\begin{array}{l}0.91 \\
(0.68-1.21)\end{array}$ & $0.98(0.74-1.30)$ & Ref & $1.21(0.93-1.57)$ & $* * * 1.72(1.34-2.22)$ \\
\hline mod inactive & 1353 & 24.7 & 374 & 24.1 & $\begin{array}{l}1.03 \\
(0.72-1.49)\end{array}$ & $1.08(0.79-1.47)$ & Ref & $1.14(0.82-1.58)$ & * $1.47(1.07-2.03)$ \\
\hline mod active & 1377 & 25.2 & 336 & 21.7 & $\begin{array}{l}0.86 \\
(0.59-1.23\end{array}$ & $0.69(0.47-1.01)$ & Ref & $0.95(0.69-1.30)$ & $1.19(0.86-1.65)$ \\
\hline active & 1295 & 23.7 & 296 & 19.1 & $\begin{array}{l}0.88 \\
(0.57-1.33)\end{array}$ & $1.01(0.69-1.49)$ & Ref & $1.22(0.87-1.70)$ & $* 1.50(1.06-2.12)$ \\
\hline \multicolumn{10}{|l|}{$\begin{array}{l}\text { By educational } \\
\text { level (1HE) }\end{array}$} \\
\hline No qualifications & 1444 & 26.4 & 537 & 34.6 & $\begin{array}{l}0.90 \\
(0.68-1.20)\end{array}$ & $1.03(0.78-1.36)$ & Ref & $0.90(0.68-1.19)$ & $* * * 1.76(1.36-2.28)$ \\
\hline O level and above & 4025 & 73.6 & 1014 & 65.4 & $\begin{array}{l}0.96 \\
(0.77-1.19)\end{array}$ & $0.90(0.73-1.11)$ & Ref & ** $1.29(1.08-1.55)$ & ** $1.38(1.14-1.67)$ \\
\hline \multicolumn{10}{|l|}{ By social class (1HE) } \\
\hline Non-manual & 3395 & 62.1 & 932 & 60.1 & $\begin{array}{l}1.03 \\
(0.82-1.29)\end{array}$ & $0.90(0.73-1.11)$ & Ref & $1.12(0.92-1.37)$ & ** $1.39(1.14-1.69)$ \\
\hline Manual & 2074 & 37.9 & 619 & 39.9 & $\begin{array}{l}0.81 \\
(0.62-1.06)\end{array}$ & $1.04(0.80-1.35)$ & Ref & $1.17(0.92-1.49)$ & $* * * 1.67(1.31-2.13)$ \\
\hline
\end{tabular}


Table 3 Cox multivariable-adjusted HRs after 16 years of follow-up for all-cause mortality in 5469 men. Results are given for stratified variables by WC change category (Continued)

\begin{tabular}{|c|c|c|c|c|c|c|c|c|c|}
\hline & & & & & \multicolumn{5}{|c|}{ Categories of change in waist circumference (WC) (cm) } \\
\hline & & & & & loss $>5 \mathrm{~cm}$ & $\begin{array}{l}\text { loss }>2.5 \\
\& \leq 5 \mathrm{~cm}\end{array}$ & $\begin{array}{l}\text { loss or gain } \\
\leq 2.5 \mathrm{~cm}\end{array}$ & $\begin{array}{l}\text { gain }>2.5 \\
\& \leq 5 \mathrm{~cm}\end{array}$ & gain $>5 \mathrm{~cm}$ \\
\hline \multicolumn{10}{|l|}{ Excluding early deaths } \\
\hline Excluding deaths $<3$ y & 5347 & 97.8 & 1429 & 92.1 & $\begin{array}{l}0.90 \\
(0.75-1.08)\end{array}$ & $\begin{array}{l}0.94 \\
(0.79-1.12)\end{array}$ & Ref & $1.16(0.99-1.35)$ & $* * * 1.52(1.29-1.78)$ \\
\hline Excluding deaths $<5$ y & 5211 & 95.3 & 1293 & 83.4 & $\begin{array}{l}0.94 \\
(0.78-1.14)\end{array}$ & $\begin{array}{l}0.94 \\
(0.79-1.13)\end{array}$ & Ref & $1.13(0.96-1.34)$ & *** $1.45(1.23-1.72)$ \\
\hline
\end{tabular}

Adjusted for age, BMI, baseline WC, physical activity, smoking, educational level, social class and change in weight (except where the variable was used for stratification)

${ }^{* * *} p<0.001 ;{ }^{* *} p<0.01 ;{ }^{*} p<0.05$

variables in men with a WCG; findings relating to WCL were inconsistent. In men who had a WCG $>5 \mathrm{~cm}$, the strongest associations were found in those who were younger, current smokers, overweight, physically active, had no qualifications, had a manual job and a normal WC. Even after excluding participants who died within 3 or 5 years of the 2HE, men with a WCG had significantly higher HRs for CVD mortality, than those in the WCM category.

\section{Total and CVD mortality: WC changes in relation to weight changes}

Weight change was strongly positively correlated with a change in WC in both men $(\mathrm{r}=0.60)$ and women $(r=$ $0.57)(P<0.001)$. We also explored the association of category changes in WC and total mortality in combination with category changes in weight; the interaction between WC change categories and weight change categories was not found to be significant ( $p=0.29$ for men and 0.82 for women). No significant interaction was found between WC change categories and weight change categories with regard to CVD mortality ( $p=$ 0.71 for men and 0.52 for women).

Figure 2a illustrates the associations between category changes in WC and weight and total mortality, adjusted for age, BMI, baseline WC, physical activity, smoking, educational level, social class and change in weight, and menopausal status in women. Similar trends in both men and women were generally evident. In men, significantly higher HRs were observed for all WTL categories, irrespective of whether men were categorised as WCL, WCM or WCG, as well as for WCG and WTM (HR 1.37 (CI 1.17-1.61)), compared to those in the WTM and WCM category. In women, significantly higher HRs were observed for total mortality for 2 categories WTL and WCL (HR 1.40 (CI 1.14-1.70)) and WTL and WCM (HR 1.50 (CI 1.16-1.95)), compared to the reference category of WTM and WCM.

Figure $2 \mathrm{~b}$ illustrates the similar associations with CVD mortality, adjusted for age, BMI, baseline WC, physical activity, smoking, educational level, social class and change in weight, and menopausal status in women. In men, significantly higher HRs were found in the WTL and WCL (HR 1.81 (CI 1.32-2.49)), WTL and WCG (HR 2.22 (CI 1.03-4.82)), and WTM and WCG (HR 1.38 (CI 1.02-1.88)) categories, compared to the reference category of WTM and WCM. A significant association for CVD mortality in women was only found in the WTL and WCM category (HR 1.81 (CI 1.15-2.85)). In all analyses, the category with the lowest HR was WTG and WCL.

\section{Discussion}

\section{Summary of main findings}

In this study of 12,337 middle-aged and elderly men and women, a WCG of more than $5 \mathrm{~cm}$ over approximately 4 years was associated with higher total mortality in both men and women and higher CVD mortality in men over the next 16 years of follow-up. Particularly among men, the associations were also evident after stratification for age, smoking, BMI, physical activity, educational level, social class and after the exclusion of deaths within the first 5 years of follow-up. Associations for WCL and mortality were inconsistent.

\section{Strengths and limitations}

The major strengths of this study include its large population of free-living, middle-aged and elderly men and women, its prospective design, and the long follow-up time. Additionally, WC, weight and height were objectively measured, not self-reported, and information on a large number of factors associated with WC and weight was available. To minimize the possibility of reverse causality, that is, participants who were sub-clinically ill were more likely to lose weight and WC which would have resulted in a higher mortality risk; participants were excluded with self-reported cancer or CVD at both time-points, as were those who had a $\mathrm{BMI}<18.5 \mathrm{~kg} / \mathrm{m}^{2}$. Those who died within the first 3 and 5 years were also excluded in our subgroup analyses. Nevertheless, we cannot completely rule out the possibility of some effect of reverse causality and/or residual confounding. 
Table 4 Cox multivariable-adjusted HRs after 16 years of follow-up for all-cause mortality in 6868 women. Results are given for stratified variables by WC change category

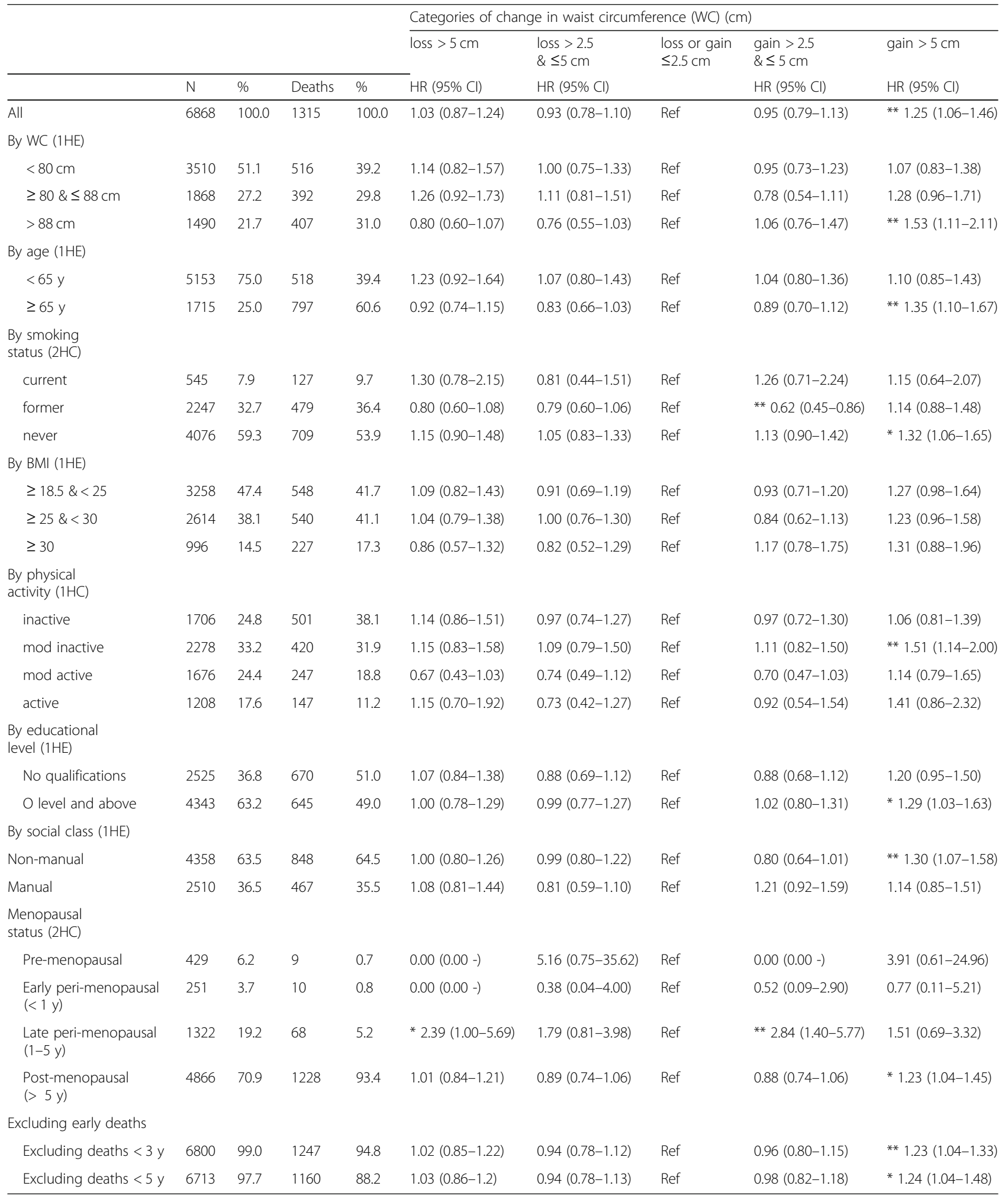

Adjusted for age, BMI, baseline WC, physical activity, smoking, educational level, social class, menopausal status and change in weight (except where the variable was used for stratification)

${ }^{* * *} p<0.001 ;{ }^{* *} p<0.01 ; * p<0.05$ 


\section{a. Total mortality in 5469 men and 6868 women by categories of change in weight and WC}

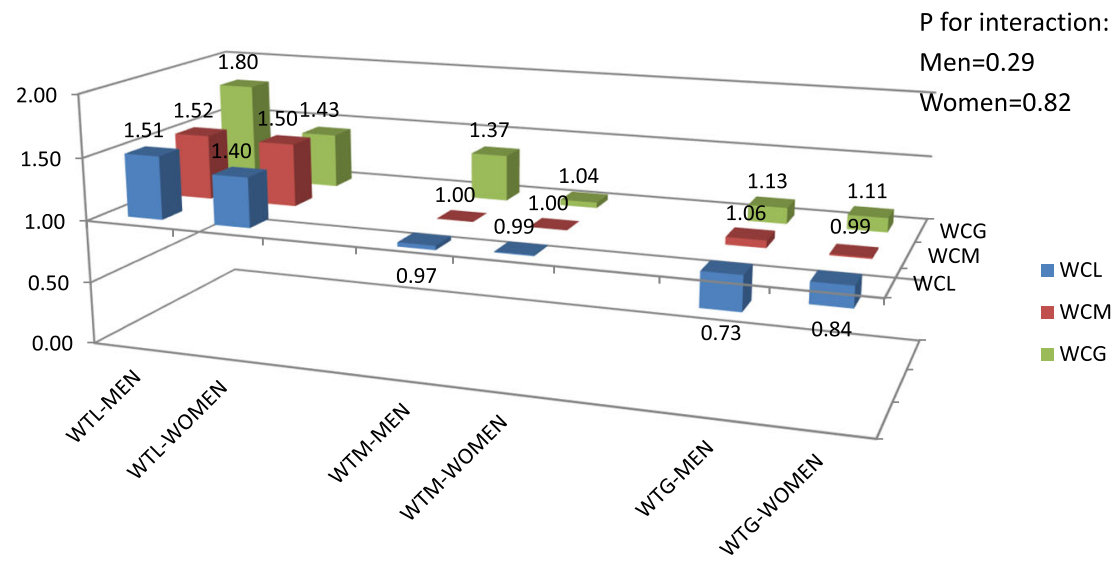

\section{b. CVD mortality in 5469 men and 6868 women by categories of change in weight and WC}

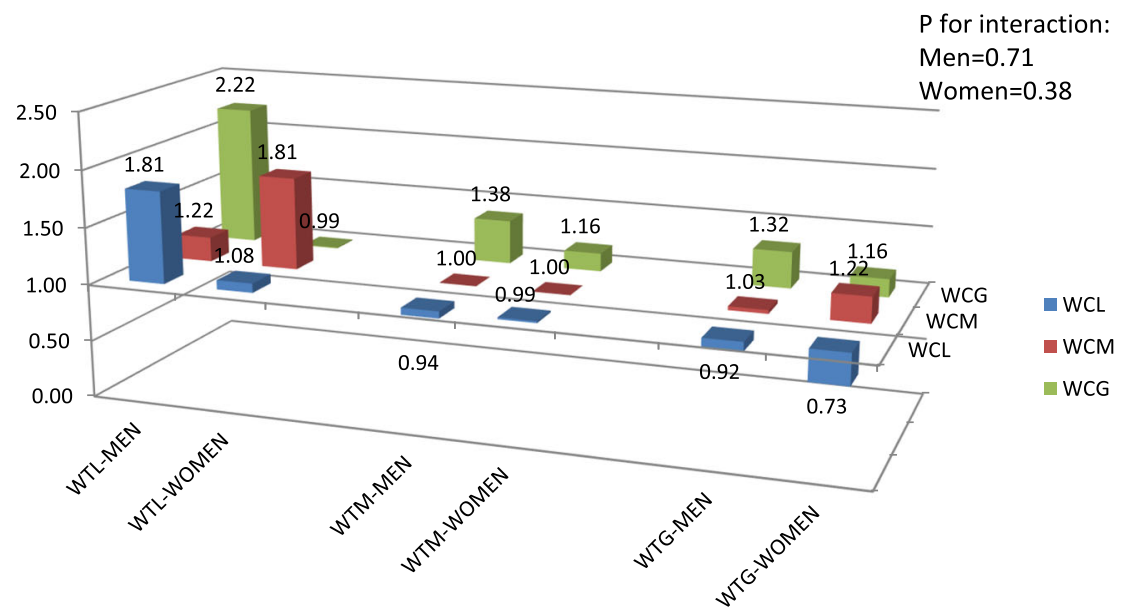

Fig 2 a Total mortality in 5469 men and 6868 women by categories of change in weight and WC, b. CVD mortality in 5469 men and 6868 women by categories of change in weight and WC

The main limitations of our study include self-reported disease history, healthy volunteer bias and attrition. The prevalence of self-reported CVD was lower in those who also attended the $2 \mathrm{HE}$, as was the percentage of current smokers and physically inactive participants, which may be indicative of healthier individuals being more likely to return for a follow up assessment (Additional file 1: Table 1). Additionally, the percentage of deaths that occurred was lower in both men and women, after exclusion criteria were applied; the percentage of deaths was highest in those who only attended the $1 \mathrm{HE}$. It is possible that some individuals in our study had undiagnosed/sub-clinical conditions such as digestive disorders, or various psychosocial diseases, such as depression, that they did not report, resulting in weight and potentially WC loss and subsequent death, and possibly an overestimation of the association [39]. The more frail participants may have been excluded and/or have not returned for the $2 \mathrm{HC}$ and therefore be under-represented in our analyses. However, it is also conceivable that some of the participants in the weight and WC loss categories were pre-frail at $2 \mathrm{HE}$. A further limitation is the inability to account for all behaviour changes during follow-up, including physical activity, which may have had effects on the observed associations.

Comparison of total and CVD mortality with other studies The highest HRs for both total and CVD mortality in men, which were statistically significant, were observed in the WTL and WCG category, although this was a small category, consisting of only 35 participants and 19 events. 
In women, the highest HR was found in the WTL and WCM category, for both total and CVD mortality. The category with the lowest HR in both men and women, for both total and CVD mortality was the weight gain and WC loss category, although this was not statistically significant. The findings that WTL, in combination with changes in WC, was associated with the highest HRs, agrees with our previous study, which found that weight loss of more than $2.5 \mathrm{~kg}$ over approximately 4 years is associated with a higher mortality over 15 years of follow-up [31] in this middle-aged and elderly population.

Our findings that WCG is associated with higher allcause mortality are in agreement with a number of previous publications. A recent pooled sample of 2492 Danish and Swedish women found that WCG over 6 years was associated with increased total and CVD mortality [23]. We did not find significant associations with WCG and CVD mortality in women; statistical power was more limited due to the lower number of CVD deaths in women in our study. In the study of Danish and Swedish women, associations were particularly strong for women with normal weight and for ever-smokers. In our analyses, we found that WCG $>5 \mathrm{~cm}$ had a similar HR for all BMI categories and only never smokers had a statistically higher HR. The differences in findings may be due to the characteristics of the 2 cohorts; our cohort of women was older, had a higher mean BMI and a lower percentage of ever-smokers. A prospective study of 26,625 healthy middle-aged men and women has also shown that increases in WC over a 5.3 year period were positively associated with mortality in men and women over 6.7 years of follow-up; the HR was 1.09 (1.02, $1,16)$ per $5 \mathrm{~cm}$ for both men and women combined, after adjusting for baseline BMI, WC and change in BMI [24].

However, results from the Melbourne Collaborative Cohort Study of 21,298 men and women aged 40-69 years at baseline found that WCL over approximately 12 years was associated with an increased risk of allcause mortality (HR: 1.26; 95\% CI: 1.09-1.47) over 7.7 years of follow-up, especially in older adults, compared to those who had minimal changes, but did not find an association with WCG [25]. It is possible that the shorter time period of less than 4 years between our WC measurements may partially explain our lack of associations with WCL and mortality. However, in a study of older adults aged $70-77$ years, WCL over a 3 year period $(\geq 3.1$ $\mathrm{cm})$ was significantly associated with an all-cause mortality risk over 20 years of follow-up of 1.52 (95\% CI: 1.01-2.31), which was comparable to their weight loss finding, in agreement with our previous results [31]; no significant associations were found for WCG and allcause mortality, or changes in WC and CVD mortality [40]. No significant associations were found with selfreported changes in WC over a 12 years and concurrent mortality, after 9 years of follow-up, in a large, prospective study among 23,254 Swedish women [26], although the cohort was relatively young (median age of 52 years at follow-up), with a relatively low number of deaths $(N=570)$ during follow-up, of which 79 were attributable to CVD. A smaller study of 1138 older adults, predominantly female, from the Bambuí (Brazil) Cohort Study of Aging [27] did not find any associations with WC change and mortality; the relatively short time interval of 3 years between measurements, in addition to the follow-up period of 8 years may have contributed to the lack of findings. No associations were found for WC change over 3.6 years and mortality in a study of 1805 Iranian men, aged $\geqslant 30$ years [28]; the limited number of deaths $(N=88)$ during the relatively short-term followup of 6.6 years resulted in limited power to assess the effect of changes in WC for mortality events.

\section{Changes in WC and weight and plausible mechanisms}

Weight, particularly in older populations, is an indicator encompassing not just fat but also bone and muscle mass and these different components may relate to health in different directions; thus, weight loss in later life may be an indicator of increasing frailty. Body fat distribution changes with age, with a reduction in subcutaneous fat and an increase in central adiposity [41]. The accumulation of body fat in the abdominal region has been shown to be associated with a number of adverse health outcomes such as diabetes, metabolic syndrome, CVD and all-cause mortality, independently of BMI [12, 42-44]. Adipose tissue has an important role to play in numerous metabolic and endocrine functions, including the expression and secretion of inflammatory cytokines, such as leptin, adiponectin, and interleukin 6 (IL-6), which are important regulatory factors of energy intake and inflammatory responses [45]. In a study of 20 abdominally obese, older women, hyperinsulinemia was positively associated with adipose IL-6 gene expression, but negatively associated with adipose adiponectin expression [46]. A 20-week weight loss program in obese, older women decreased leptin production in both gluteal and abdominal adipose tissue, but only increased adiponectin production from abdominal adipose tissue [47]. These results highlight the importance that regional adipose tissue hormone/cytokine production may play in mechanisms of metabolic complications associated with abdominal obesity and the importance of improving body fat distribution, through appropriate exercise and diet programs [48].

\section{Public health considerations}

Obesity is a complex condition, for which there are numerous risk factors, many of which are modifiable $[49,50]$. Attention needs to be focussed on various levels of policy strategies for its prevention [51,52]. Aa a number of studies 
have shown that WC is increasing at a faster rate than BMI or body weight [53-55], current recommendations, based on observational cohort studies, including findings from this publication, should advocate prevention of WC gain into adulthood, and weight and WC stability from midlife onwards.

\section{Conclusion}

In summary, an increase in WC of more than $5 \mathrm{~cm}$ over approximately 4 years, with little weight gain, is significantly associated with higher total mortality in both men and women and higher CVD mortality in men over the next 16 years of follow-up in this population-based cohort study of 12,337 middle-aged and elderly men and women. These findings are in marked contrast to our earlier observation that weight loss is associated with increased mortality risk [31]. The apparently paradoxical observations suggest that WC may be a better indicator of the adverse health consequence of obesity in later life than weight. Interventions targeted at fat distribution and focusing on preventing increase in central adiposity rather than lowering weight per se in later life may be more likely to have health benefits.

\section{Supplementary information}

Supplementary information accompanies this paper at https://doi.org/10. 1186/s12872-019-1223-z

Additional file 1. Supplementary information. Baseline characteristics of EPIC-Norfolk men and women who attended $1 \mathrm{HE}$, and those who attended both $1 \mathrm{HE}$ and $2 \mathrm{HE}$, before and after exclusion criteria were applied. Cox multivariable-adjusted HRs after 16 years of follow-up for CVD mortality in 5469 men

\section{Abbreviations}

1HE: Baseline health examination; 2HE: Second health examination; BMI: Body mass index; CVD: Cardiovascular disease; EPIC: European prospective investigation into Cancer and nutrition; WC: Waist circumference; WCG: Waist circumference gain; WCL: Waist circumference loss; WCM: Waist circumference maintenance; WTG: Weight gain; WTL: Weight loss; WTM: Weight maintenance

\section{Acknowledgements}

We gratefully thank all the EPIC-Norfolk participants and collaborating GP practices in Norfolk, as without their contribution, this study would not have been possible. We would also like to thank all the staff in Norfolk and Cambridge for their dedication and hard work.

\section{Authors' contributions}

AAM wrote the manuscript and analysed the data. KTK and MAHL advised on statistical analysis. RL manages the study data. KTK and NW are principal investigators who contributed to the conception and study design and guidance on the presentation and interpretation of these data. All authors contributed to data analysis and interpretation of the data, and all authors reviewed and approved the final manuscript.

\section{Funding}

This work was supported by the Medical Research Council, UK (G9502233) and Cancer Research UK (CRUK SP2024/0201, SP2024/0204). None of the funding organisations had a role in the design or conduct of this research.

\section{Availability of data and materials}

The datasets analysed during the current study are available from the corresponding author on reasonable request.

\section{Ethics approval and consent to participate}

The study was approved by the Norfolk District Health Authority Ethics Committee and all participants gave written, informed consent.

Consent for publication

Not applicable.

\section{Competing interests}

The authors declare that they have no competing interests.

\section{Author details}

${ }^{1}$ European Prospective Investigation into Cancer and Nutrition, Department of Public Health and Primary Care, Strangeways Research Laboratory, University of Cambridge, Worts Causeway, Cambridge, UK. ${ }^{2}$ European Prospective Investigation into Cancer and Nutrition, MRC Epidemiology Unit, Cambridge Biomedical Campus, University of Cambridge, Cambridge, UK ${ }^{3}$ MRC Epidemiology Unit, Cambridge, Institute of Metabolic Science, Cambridge Biomedical Campus, University of Cambridge School of Clinical Medicine, Cambridge, UK. 'EPIC, Department of Gerontology, Addenbrooke's Hospital, School of Clinical Medicine, University of Cambridge, Cambridge, UK

Received: 11 April 2019 Accepted: 10 October 2019

Published online: 28 October 2019

\section{References}

1. Bauer UE, Briss PA, Goodman RA, Bowman BA. Prevention of chronic disease in the 21st century: elimination of the leading preventable causes of premature death and disability in the USA. Lancet [Internet]. 2014:384(9937): 45-52 Available from: https://doi.org/10.1016/S0140-6736(14)60648-6.

2. Global Burden of Metabolic Risk Factors for Chronic Diseases Collaboration (BMI Mediated Effects), Lu Y, Hajifathalian K, Ezzati M, Woodward M, Rimm EB, Danaei G. Metabolic mediators of the effects of body-mass index, overweight, and obesity on coronary heart disease and stroke: a pooled analysis of 97 prospective cohorts with 1.8 million participants. Lancet. 2014 383(9921):970-83.

3. Wang H, Naghavi M, Allen C, Barber RM, Carter A, Casey DC, et al. Global, regional, and national life expectancy, all-cause mortality, and cause-specific mortality for 249 causes of death, 1980-2015: a systematic analysis for the global burden of disease study 2015. Lancet. 2016;388(10053):1459-544.

4. WHO. Physical status: the use and interpretation of anthropometry. Report of a WHO Expert Committee. Vol. 854, World Health Organization technical report series. 1995. p. 1-452.

5. St-Onge MP, Gallagher D. Body composition changes with aging: the cause or the result of alterations in metabolic rate and macronutrient oxidation? Nutrition [Internet]. 2010;26(2):152-5 Available from: https://doi.org/10.1016/ j.nut.2009.07.004.

6. Janssen I, Heymsfield Steven B, Allison David B, Kotler Donald P, Ross R. Body mass index and waist circumference independently ontribute to the prediction of nonabdominal, abdominal subcutaneous and visceral fat. Am J Clin Nutr. 2002;75(May):683-8.

7. Snijder MB, van Dam RM, Visser M, Seidell JC. What aspects of body fat are particularly hazardous and how do we measure them? Int J Epidemiol. 2006;35(1):83-92.

8. Lakka HM, Lakka TA, Tuomilehto J, Salonen JT. Abdominal obesity is associated with increased risk of acute coronary events in men. Eur Heart J. 2002;23(9):706-13.

9. Zhu S, Wang Z, Heshka S, Heo M, Faith MS, Heymsfield SB. Waist circumference and obesity-associated risk factors among whites in the third National Health and Nutrition Examination. 2002;

10. Zhu S, Heshka S, Wang ZM, Shen W, Allison DB, Ross R, et al. Combination of BMI and waist circumference for identifying cardiovascular risk factors in whites. Obes Res. 2004;12(4):633-45.

11. Czernichow S, Kengne A-P, Stamatakis E, Hamer M, Batty GD. Body mass index, waist circumference and waist-hip ratio: which is the better discriminator of cardiovascular disease mortality risk? Evidence from an individual-participant meta-analysis of 82864 participants from nine 
cohort studies. Obes Rev [Internet]. 2011;(6):no-no. Available from: http://doi. wiley.com/10.1111/j.1467-789X.2011.00879.x

12. Pischon T, Boeing H, Hoffmann K, Bergmann M, Schulze MB, Overvad K, et al. General and abdominal adiposity and risk of death in Europe. N Engl J Med [Internet]. 2008;359(20):2105-20 Available from: http://www.nejm.org/ doi/abs/10.1056/NEJMoa0801891. Accessed 19 June 2018.

13. Folsom AR, Kushi LH, Anderson KE, Mink PJ, Olson JE, Hong C-P, et al. Associations of General and Abdominal Obesity With Multiple Health Outcomes in Older Women. Arch Intern Med [Internet]. 2000;160(14):2117 Available from: http://archinte.jamanetwork.com/article.aspx?doi=10.1001/ archinte.160.14.2117. Accessed 19 June 2018

14. Janssen I, Katzmarzyk PT, Ross R. Body mass index is inversely related to mortality in older people after adjustment for waist circumference. J Am Geriatr Soc. 2005;53(12):2112-8

15. Simpson JA, Maclnnis RJ, Peeters A, Hopper JL, Giles GG, English DR. A comparison of adiposity measures as predictors of all-cause mortality: the Melbourne collaborative cohort study. Obesity. 2007;15(4):994-1003.

16. Zhang C, Rexrode KM, Van Dam RM, Li TY, Hu FB. Abdominal obesity and the risk of all-cause, cardiovascular, and cancer mortality: sixteen years of follow-up in US women. Circulation. 2008;117(13):1658-67.

17. Koster A, Leitzmann MF, Schatzkin A, Mouw T, Adams KF, van Eijk JTM, et al, Waist circumference and mortality. Am J Epidemiol. 2008;167(12):1465-75.

18. Jacobs EJ. Waist Circumference and All-Cause Mortality in a Large US Cohort. Arch Intern Med [Internet]. 2010;170(15):1293 Available from: http:// archinte.jamanetwork.com/article.aspx?doi=10.1001/archinternmed.2010.201. Accessed 22 May 2018.

19. Price GM, Uauy R, Breeze E, Bulpitt CJ, Fletcher AE. Weight, shape, and mortality risk in older persons : elevated waist- hip ratio, not high body mass index, is associated with a greater risk. 2006;

20. Walter S, Kunst A, MacKenbach J, Hofman A, Tiemeier H. Mortality and disability: the effect of overweight and obesity. Int J Obes [Internet]. 2009; 33(12):1410-8 Available from: https://doi.org/10.1038/ijo.2009.176.

21. Grundy SM, Cleeman JI, Daniels SR, Donato KA, Eckel RH, Franklin BA, et al. Diagnosis and management of the metabolic syndrome: an American Heart Association/National Heart, Lung, and Blood Institute scientific statement. Circulation. 2005;112(17):2735-52

22. O'Neill S, O'Driscoll L. Metabolic syndrome: a closer look at the growing epidemic and its associated pathologies. Obes Rev. 2015;16(1):1-12.

23. Klingberg S, Mehlig K, Lanfer A, Björkelund C, Heitmann BL, Lissner L. Increase in waist circumference over 6 years predicts subsequent cardiovascular disease and total mortality in nordic women. Obesity [Internet] 2015;23(10):2123-2130. Available from: http://doi.wiley.com/10.1002/oby.21203

24. Berentzen TL, Jakobsen MU, Halkjaer J, Tjønneland A, Overvad K, Sørensen TI. a. Changes in waist circumference and mortality in middle-aged men and women. PLoS One. 2010;5(9):1-8.

25. Karahalios A, Simpson J, Baglietto L, Maclnnis RJ, Hodge AM, Giles GG, et al. Change in body size and mortality: results from the Melbourne collaborative cohort study. PLoS One. 2014;9(7):e99672.

26. Roswall N, Li Y, Sandin S, Ström P, Adami HO, Weiderpass E. Changes in body mass index and waist circumference and concurrent mortality among Swedish women. Obesity. 2017:25(1):215-22.

27. Beleigoli AM, Diniz MDFH, Boersma E, Silva JL, Lima-Costa MF, Ribeiro AL. The effects of weight and waist change on the risk of long-term mortality in older adults- the Bambuí (Brazil) cohort study of aging. J Nutr Health Aging [Internet]. 2017;21 (8):861-6 Available from: http://link.springer.com/1 0.1007/s12603-016-0858-z. Accessed 22 May 2018.

28. Mousavi SV, Mohebi R, Mozaffary A, Sheikholeslami F, Azizi F, Hadaegh F. Changes in body mass index, waist and hip circumferences, waist to hip ratio and risk of all-cause mortality in men. Eur J Clin Nutr [Internet]. 2015; 69(8):927-32 Available from: https://doi.org/10.1038/ejcn.2014.235.

29. De Koning L, Merchant AT, Pogue J, Anand SS. Waist circumference and waist-to-hip ratio as predictors of cardiovascular events: meta-regression analysis of prospective studies. Eur Heart J. 2007;28(7):850-6.

30. NatCen Social Research. Health Survey for England 2016 [Internet]. 2017. 143 p. Available from: https://digital.nhs.uk/catalogue/PUB30169. Accessed 30 Apr 2018.

31. Mulligan AA, Lentjes MAH, Luben RN, Wareham NJ, Khaw K-T. Weight change and 15 year mortality: results from the European prospective investigation into Cancer in Norfolk (EPIC-Norfolk) cohort study. Eur J Epidemiol [Internet]. 2017:33(1):41-57 Available from: http://link.springer. com/10.1007/s10654-017-0343-y. Accessed 10 July 2018.
32. Riboli E. Nutrition and cancer : background and rationale of the European prospective investigation into Cancer and nutrition ( EPIC ). Ann Oncol. 1992;3:783-91.

33. Day N, Oakes S, Luben R, Khaw KT, Bingham S, Welch A, et al. EPIC-Norfolk: study design and characteristics of the cohort. European Prospective Investigation of Cancer. Br J Cancer. 1999 Jul;80(Suppl 1):95-103.

34. Bennett N, Dodd T, Flatley J, BK FS. Health survey for England 1993. London: HMSO; 1995.

35. Wareham NJ, Jakes RW, Rennie KL, Schuit J, Mitchell J, Hennings S, et al. Validity and repeatability of a simple index derived from the short physical activity questionnaire used in the European prospective investigation into Cancer and nutrition (EPIC) study. Public Health Nutr. 2003 Jun;6(4):407-13.

36. Khaw K-T, Jakes R, Bingham S, Welch A, Luben R, Day N, et al. Work and leisure time physical activity assessed using a simple, pragmatic, validated questionnaire and incident cardiovascular disease and all-cause mortality in men and women: The European Prospective Investigation into Cancer in Norfolk prospective pop. Int J Epidemiol [Internet]. 2006:35(4):1034-43 Available from: https:/www.scopus.com/inward/record.url?eid=2-s2.0-3374 9588206\&partner $\mid \mathrm{D}=40 \& \mathrm{md} 5=5$ fef3b04de9078807d2c6a2788be2f20. Accessed 20 June 2018.

37. Shohaimi S, Luben R, Wareham N, Day N, Bingham S, Welch A, et al. Residential area deprivation predicts smoking habit independently of individual educational level and occupational social class. A cross sectional study in the Norfolk cohort of the European investigation into Cancer (EPICNorfolk). J Epidemiol Community Health. 2003;57(3):270-6.

38. UNESCO. International Standard Classification of Education ISCED 1997 [Internet]. 1997 [cited 2017 Oct 10]. Available from: http://www.unesco.org/ education/information/nfsunesco/doc/isced_1997.htm. Accessed 11 Sept 2018

39. Bosch X, Monclús E, Escoda O, Guerra-García M, Moreno P, Guasch N, et al. Unintentional weight loss: clinical characteristics and outcomes in a prospective cohort of 2677 patients. PLoS One. 2017;12(4):1-20

40. de Hollander EL, Bemelmans WJE, de Groot LCPGM. Associations between changes in anthropometric measures and mortality in old age: a role for mid-upper arm circumference? J Am Med Dir Assoc [Internet]. 2013;14(3): 187-93 Available from: http://linkinghub.elsevier.com/retrieve/pii/S1525861 012003465. Accessed 7 Aug 2018

41. Kuk JL, Saunders TJ, Davidson LE, Ross R. Age-related changes in total and regional fat distribution. Ageing Res Rev. 2009;8(4):339-48.

42. Janssen I, Katzmarzyk PT, Ross R. Waist circumference and not body mass index explains obesity- related health risk. Am J Clin Nutr. 2004;79:379-84.

43. Suk S-H, Sacco RL, Boden-Albala B, Cheun JF, Pittman JG, Elkind MS, et al. Abdominal obesity and risk of ischemic stroke. Stroke [Internet]. 2003;34(7): 1586-92 Available from: https://www.ahajournals.org/doi/10.1161/01.STR. 0000075294.98582.2F. Accessed 19 June 2018.

44. Balkau B, Deanfield JE, Després J-P, Bassand J-P, Fox KAA, Smith SC, et al. International Day for the evaluation of abdominal obesity (IDEA). Circulation [Internet]. 2007;116(17):1942-51 Available from: https://www.ahajournals. org/doi/10.1161/CIRCULATIONAHA.106.676379. Accessed 11 Sept 2018.

45. Kershaw EE, Flier JS. Adipose tissue as an endocrine organ. J Clin Endocrinol Metab. 2004;89(6):2548-56.

46. You T, Yang R, Lyles MF, Gong D, Nicklas BJ. Abdominal adipose tissue cytokine gene expression: relationship to obesity and metabolic risk factors. Am J Physiol Metab [Internet]. 2005;288(4): E741-7 Available from: http://www.nutritionjrnl.com/article/ S0899900707001839/fulltext\%5Cn, http://www.biomedcentral.com/14 71-2458/10/158/abstract\%5Cn, http://www.biomedcentral.com/14 71-2458/10/158\%5Cn, http://www.biomedcentral.com/content/pdf/14 71-2458-10-158.pdf\%5Cn, http://www.scien. Accessed 7 Aug 2018.

47. You T, Wang X, Murphy KM, Lyles MF, Demons JL, Yang R, et al. Regional adipose tissue hormone/cytokine production before and after weight loss in abdominally obese women. Obesity. 2014;22(7):1679-84.

48. Arciero P, Gentile C, Martin-Pressman R, Ormsbee M, Everett M, Zwicky L, et al. Increased dietary protein and combined high intensity aerobic and resistance exercise improves body fat distribution and cardiovascular risk factors. Int J Sport Nutr Exerc Metab. 2006;16(4):373-92.

49. Seidell JC, Halberstadt J. The global burden of obesity and the challenges of prevention. Ann Nutr Metab. 2015;66(suppl 2):7-12.

50. Hruby A, Hu FB. The epidemiology of obesity: a big picture. Pharmacoeconomics [Internet]. 2015;33(7):673-89 Available from: https:// doi.org/10.1007/s40273-014-0243-x. 
51. Malik VS, Willett WC, Hu FB. Global obesity: trends, risk factors and policy implications. Nat Rev Endocrinol [Internet]. 2013;9(1):13-27 Available from: https://doi.org/10.1038/nrendo.2012.199.

52. Malik VS, Hu FB. Obesity Prevention. In: Prabhakaran D, Anand S, Gaziano TA, Mbanya J-C, Wu Y, Nugent R, editors. Disease Control Priorities, Third Edition (Volume 5): Cardiovascular, Respiratory, and Related Disorders [Internet]. 3rd ed. The World Bank; 2017. p. 117-34. Available from: http:// elibrary.worldbank.org/doi/book/10.1596/978-1-4648-0518-9. Accessed 12 Sept 2018.

53. Walls HL, Stevenson CE, Mannan HR, Abdullah A, Reid CM, McNeil JJ, et al. Comparing trends in BMl and waist circumference. Obesity [Internet]. 2011; 19(1):216-9 Available from: https://doi.org/10.1038/oby.2010.149.

54. Freedman DS, Ford ES. Are the recent secular increases in the waist circumference of adults independent of changes in BMI? Am J Clin Nutr [Internet] 2015;101(3):425-431. Available from: https://academic.oup.com/ ajcn/article/101/3/425/4569394. Accessed 12 Sept 2018.

55. Albrecht SS, Gordon-Larsen P, Stern D, Popkin BM. Is waist circumference per body mass index rising differentially across the United States, England, China and Mexico? Eur J Clin Nutr [Internet]. 2015;69(12):1306-12 Available from: https://doi.org/10.1038/ejen.2015.71. Accessed 12 Sept 2018.

\section{Publisher's Note}

Springer Nature remains neutral with regard to jurisdictional claims in published maps and institutional affiliations.

Ready to submit your research? Choose BMC and benefit from:

- fast, convenient online submission

- thorough peer review by experienced researchers in your field

- rapid publication on acceptance

- support for research data, including large and complex data types

- gold Open Access which fosters wider collaboration and increased citations

- maximum visibility for your research: over $100 \mathrm{M}$ website views per year

At BMC, research is always in progress.

Learn more biomedcentral.com/submissions 